\title{
Classical and Quantum Algorithms for Constructing Text from Dictionary Problem
}

\author{
Kamil Khadiev $^{1,2, \star}$ and Vladislav Remidovskii ${ }^{2}$ \\ ${ }^{1}$ Smart Quantum Technologies Ltd., Kazan, Russia \\ ${ }^{2}$ Kazan Federal University, Kazan, Russia \\ kamilhadi@gmail.com,vladremidovskiyvs@gmail.com
}

\begin{abstract}
We study algorithms for solving the problem of constructing a text (long string) from a dictionary (sequence of small strings). The problem has an application in bioinformatics and has a connection with the Sequence assembly method for reconstructing a long DNA sequence from small fragments. The problem is constructing a string $t$ of length $n$ from strings $s^{1}, \ldots, s^{m}$ with possible intersections. We provide a classical algorithm with running time $O\left(n+L+m(\log n)^{2}\right)=\tilde{O}(n+L)$ where $L$ is the sum of lengths of $s^{1}, \ldots, s^{m}$. We provide a quantum algorithm with running time $O(n+\log n \cdot(\log m+\log \log n) \cdot \sqrt{m \cdot L})=\tilde{O}(n+\sqrt{m \cdot L})$. Additionally, we show that the lower bound for the classical algorithm is $\Omega(n+L)$. Thus, our classical algorithm is optimal up to a log factor, and our quantum algorithm shows speed-up comparing to any classical algorithm in a case of non-constant length of strings in the dictionary.
\end{abstract}

Keywords: quantum computation, quantum models, quantum algorithm, query model, string constructing, sequence assembly, DNA constructing

\section{Introduction}

Quantum computing $[18,2,1]$ is one of the hot topics in computer science of last decades. There are many problems where quantum algorithms outperform the best known classical algorithms $[6,8,12,11]$.

One of such problems are problems for strings. Researchers show the power of quantum algorithms for such problems in $[16,4,19,10]$.

In this paper, we consider the problem of constructing text from dictionary strings with possible intersections. We have a text $t$ of length $n$ and a dictionary $s=$ $s^{1}, \ldots, s^{m}$. The problem is constricting $t$ only from strings of $s$ with possible intersections. The problem is connected with the sequence assembly method for reconstructing a long DNA sequence from small fragments [17,3].

We suggest a classical algorithm with running time $O\left(n+L+m(\log n)^{2}\right)=$ $\tilde{O}(n+L)$, where $L=\left|s^{1}\right|+\cdots+\left|s^{m}\right|,\left|s^{i}\right|$ is a length of $s^{i}$ and $\tilde{O}$ does not consider $\log$ factors. The algorithm uses segment tree[13] and suffix array[15] data structures, concepts of comparing string using rolling hash $[9,7]$ and idea of prefix sum [5].

The second algorithm is quantum. It uses similar ideas and quantum algorithm for comparing two strings with quadratic speed-up comparing to classical counterparts [10]. The running time for our quantum algorithm is

$$
O(n+\log n \cdot(\log m+\log \log n) \cdot \sqrt{m \cdot L})=\tilde{O}(n+\sqrt{m \cdot L}) .
$$

^ This work was supported by Russian Science Foundation Grant 19-71-00149. 
Additionally, we show the lower bound in a classical case that is $\Omega(n+L)$. Thus, we get the optimal classical algorithm in a case of $m(\log n)^{2}=O(L+n)$. It is true, for example, in a case of $O(m)$ strings form $s$ has length at least $\Omega\left((\log n)^{2}\right)$ or in a case of $m=o\left(n /(\log n)^{2}\right)$. In the general case, the algorithm is an optimal algorithm up to a log factor. The quantum algorithm is better than any classical counterparts in a case of $\log n \cdot(\log m+\log \log n) \cdot \sqrt{m \cdot L}=o(L)$. It happens if $O(m)$ strings from $s$ has length at least $\Omega(\log n \cdot(\log m+\log \log n))$.

Our algorithm uses some quantum algorithms as a subroutine, and the rest part is classical. We investigate the problems in terms of query complexity. The query model is one of the most popular in the case of quantum algorithms. Such algorithms can do a query to a black box that has access to the sequence of strings. As a running time of an algorithm, we mean a number of queries to the black box.

The structure of the paper is the following. We present tools in Section 2. Then, we discuss the classical algorithm in Section 3.1 and quantum algorithm in Section 3.2. Section 4 contains lower bound.

\section{Tools}

Our algorithms uses several data structures and algorithmic ideas like segment tree[13], suffix array[15], rolling hash[9] and prefix sum [5]. Let us describe them in this section.

\subsection{Preliminaries}

Let us consider a string $u=\left(u_{1}, \ldots, u_{l}\right)$ for some integer $l$. Then, $|u|=l$ is the length of the string. $u[i, j]=\left(u_{i}, \ldots, u_{j}\right)$ is a substring of $u$.

In the paper, we compare strings in lexicographical order. For two strings $u$ and $v$, the notation $u<v$ means $u$ precedes $v$ in lexicographical order.

\subsection{Rolling Hash for Strings Comparing}

The rolling hash was presented in [9]. It is a hash function

$$
h_{p}(u)=\left(\sum_{i=1}^{|u|} \operatorname{index}\left(u_{i}\right) \cdot K^{i-1}\right) \bmod p,
$$

where $p$ is some prime integer, $K=|\Sigma|$ is a size of the alphabet and index $\left(u_{i}\right) \in$ $\{0, \ldots, K-1\}$ is the index of a symbol $u_{i}$ in the alphabet. For simplicity we consider binary alphabet. So, $K=2$ and $i n \operatorname{dex}\left(u_{i}\right)=u_{i}$.

We can use rolling hash and the fingerprinting method [7] for comparing two strings $u, v$. Let us randomly choose $p$ from the set of the first $r$ primes, such that $r \leq$ $\frac{\max (|u|,|v|)}{\varepsilon}$ for some $\varepsilon>0$. Due to Chinese Theorem and [7], the following statements are equivalent $h_{p}(u)=h_{p}(v)$ and $u=v$ with error probability at most $\varepsilon$. If we have $\delta$ invocations of comparing procedure, then we should choose $\frac{\delta \cdot \max (|u|,|v|)}{\varepsilon}$ primes. Due to Chebishev's theorem, the $r$-th prime number $p_{r} \approx r \ln r$. So, if our data type for integers is enough for storing $\frac{\delta \cdot \max (|u|,|v|)}{\varepsilon} \cdot(\ln (\delta)+\ln (\max (|u|,|v|))-\ln (\varepsilon))$, then it is enough for computing the rolling hash. 
Additionally, for a string $u$, we can compute prefix rolling hash, that is $h_{p}(u[1, i])$. It can be computed in $O(|u|)$ running time using formula

$$
h_{p}(u[1, i])=\left(h_{p}(u[1, i-1])+\left(2^{i-1} \bmod p\right) \cdot u_{i}\right) \bmod p \text { and } h_{p}(u[1: 0])=0 .
$$

Assume, that we store $\mathcal{K}_{i}=2^{i-1} \bmod p$. We can compute all of them in $O(|u|)$ running time using formula $\mathcal{K}_{i}=\mathcal{K}_{i-1} \cdot 2 \bmod p$.

Using precomputed prefix rolling hash for a string $u$ we can compute rolling hash for any substring $u[i, j]$ in $O(1)$ running time by formula

$$
\begin{aligned}
h_{p}(u[i, j])= & \left(\sum_{q=j}^{i} u_{q} \cdot 2^{q-1-(j-1)}\right) \bmod p=\left(\sum_{q=j}^{i} u_{q} \cdot 2^{q-1}\right) \cdot 2^{-(j-1)} \bmod p= \\
= & \left(\left(\sum_{q=1}^{i} u_{q} \cdot 2^{q-1}\right)-\left(\sum_{q=1}^{j-1} u_{q} \cdot 2^{q-1}\right)\right) \cdot 2^{-(j-1)} \bmod p= \\
& =\left(h_{p}(u[1, i])-h_{p}(u[1, j-1])\right) \cdot\left(2^{-(j-1)}\right)(\bmod p) .
\end{aligned}
$$

For computing the formula in $O(1)$ we should precompute $\mathcal{I}_{i}=2^{-i} \bmod p$. We can compute it in $O(\log p+|u|)$ by the formula $\mathcal{I}_{i}=\mathcal{I}_{i-1} \cdot 2^{-1} \bmod p$ and $\mathcal{I}_{0}=1$. Due to Fermat's little theorem $2^{-1} \bmod p=2^{p-2} \bmod p$. We can compute it with $O(\log p)$ running time using Exponentiation by squaring algorithm.

Let $\operatorname{ComputeKI}(\beta, p)$ be a procedure that computes $\mathcal{K}$ and $\mathcal{I}$ up to the power $\beta$ with $O(\beta+\log p)$ running time. Let $\operatorname{ComputePrefixRollingHashes}(u, p)$ be a procedure that computes all prefix rolling hashes for a string $u$ and store them.

Assume, that we have two strings $u$ and $v$ and already computed prefix rolling hashes. Then, we can compare these strings in lexicographical order in $O(\log \min (|u|,|v|))$ running time. The algorithm is following. We search the longest common prefix of $u$ and $v$, that is $l c p(u, v)$. We can do it using binary search.

- If a mid $\leq l c p(u, v)$, then $h_{p}(u[1, m i d])=h_{p}(v[1, m i d])$.

- If a mid $>l c p(u, v)$, then $h_{p}(u[1, m i d]) \neq h_{p}(v[1, m i d])$.

Using binary search we find the last index $x$ such that $h_{p}(u[1, x])=h_{p}(v[1, x])$ and $h_{p}(u[1, x+1]) \neq h_{p}(v[1, x+1])$. In that case $l c p(u, v)=x$

After that, we compare $u_{t}$ and $v_{t}$ for $t=l c p(u, v)+1$. If $u_{t}<v_{t}$, then $u<v$; if $u_{t}>v_{t}$, then $u>v$; if $|u|=|v|=l c p(u, v)$, then $u=v$.

Binary search works with $O(\log (\min (|u|,|v|)))$ running time because we have computed all prefix rolling hashes already.

Let $\operatorname{Compare}(u, v)$ be a procedure that compares $u$ and $v$ and returns -1 if $u<v ; 1$ if $u>v$; and 0 if $u=v$.

\subsection{Segment Tree with Range Updates}

We consider a standard segment tree data structure [13] for an array $a=\left(a_{1}, \ldots, a_{l}\right)$ for some integer $l$. A segment tree for and array a can be constructed in $O(l)$ running time. The data structure allows us to invoke the following requests in $O(\log l)$ running time. 
- Update. Parameters are three integers $i, j, x(1 \leq i \leq j \leq l)$. We assign $a_{q}=$ $\max \left(a_{q}, x\right)$ for $i \leq q \leq j$.

- Push. We push all existing range updates.

- Request. For an integer $i(1 \leq i \leq l)$, we should return $a_{i}$.

Let ConstructSegmentTree $(a)$ be a function that constructs and returns a segment tree for an array $a$ in $O(l)$ running time.

Let $\operatorname{Update}(s t, i, j, x)$ be a procedure that updates a segment tree $s t$ in $O(\log l)$ running time.

Let $\operatorname{Push}(s t)$ be a procedure that push all existing range updates for a segment tree st in $O(l)$ running time.

Let $\operatorname{Request}(s t, i)$ be a function that returns $a_{i}$ from a segment tree st. The running time of the procedure is $O(\log l)$. At the same time, if we invoke PusH procedure and after that do not invoke UPDATE procedure, then the running time of REQUEST is $O(1)$.

\subsection{Suffix Array}

Suffix array [15] is an array suf $=\left(s u f_{1}, \ldots, s u f_{l}\right)$ for a string $u$ and $l=|u|$. The suffix array is a lexicographical order for all suffixes of $u$. Formally, $u\left[s u f_{i}, l\right]<u\left[s u f_{i+1}, l\right]$ for any $i \in\{1, \ldots, l-1\}$.

The suffix array can be computed in $O(l)$ running time.

Lemma 1 ([14]). A suffix array for a string u can be constructed in $O(|u|)$ running time.

Let ConstructSuffixArray $(u)$ be a procedure that constructs a suffix array for a string $u$.

\section{Algorithms}

Let us formally present the problem.

Problem. For some positive integers $n$ and $m$, we have a sequence of strings $s=\left(s^{1}, \ldots, s^{m}\right)$. Each $s^{i}=\left(s_{1}^{i}, \ldots, s_{l}^{i}\right) \in \Sigma^{l}$ where $\Sigma$ is some finite size alphabet and $l=\left|s^{i}\right|$. We call $s$ dictionary. Additionally, we have a string $t$ of length $n$, where $t=\left(t_{1}, \ldots, t_{n}\right) \in \Sigma^{n}$. We call $t$ text. The problem is searching a subsequence $s^{i_{1}}, \ldots, s^{i_{z}}$ and positions $q_{1}, \ldots, q_{z}$ such that $q_{1}=1, q_{z}=n-\left|s^{i_{z}}\right|+1, q_{j} \leq q_{j-1}+\left|s^{i_{j-1}}\right|$ for $j \in\{2, \ldots, z\}$. Additionally, $t\left[q_{j}, q_{j}+\left|s^{i_{j}}\right|-1\right]=s^{i_{j}}$ for $j \in\{1, \ldots, z\}$.

For simplicity, we assume that $\Sigma=\{0,1\}$, but all results are right for any finite alphabet.

Informally, we want to construct $t$ from $s$ with possible intersections.

Firstly, let us present a classical algorithm.

\subsection{A Classical Algorithm}

Let us present the algorithm. Let $l o n g_{i}$ be an index of a longest string from $s$ that can start in position $i$. Formally, $\operatorname{long}_{i}=j$ if $s^{j}$ is a longest string from $s$ such that $t\left[i, i+\left|s^{j}\right|-1\right]=s^{j}$. Let $\operatorname{long}_{i}=-1$ if there is no such string $s^{j}$. If we construct such 


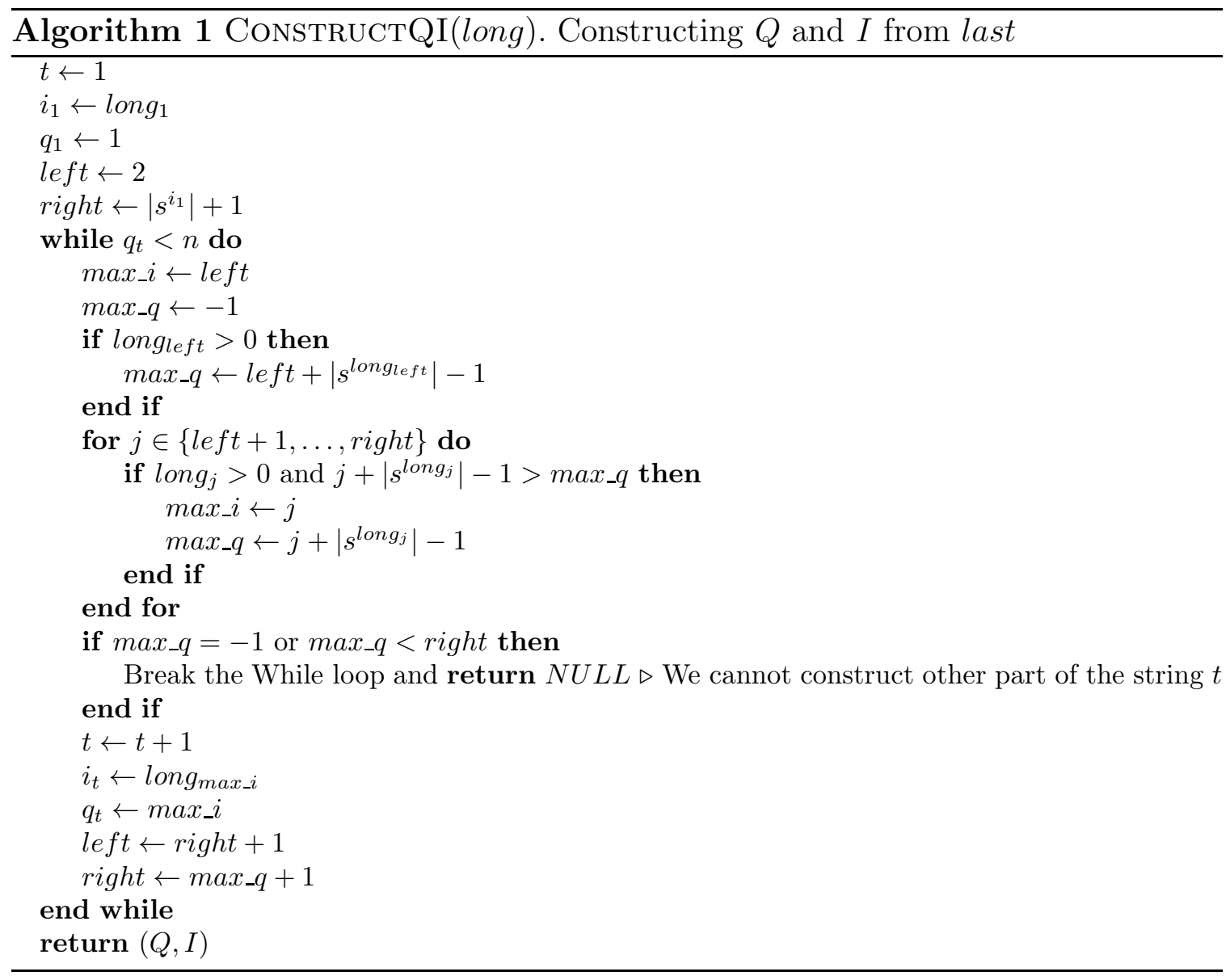


array, then we can construct $Q=\left(q_{1}, \ldots, q_{z}\right)$ and $I=\left(i_{1}, \ldots, i_{z}\right)$ that is solution of the problem in $O(n)$. A procedure ConstructQI(long) from Algorithm 1 shows it. If there is no such decomposition of $t$, then the procedure returns $N U L L$.

Let us discuss how to construct long array.

As a first step, we choose a prime $p$ that is used for rolling hash. We choose $p$ randomly from the first $z=n \cdot m \cdot 4\left\lceil\log _{2} n\right\rceil^{2} \cdot \frac{1}{\varepsilon}$ primes. In that case, due to results from Section 2.2, the probability of error is at most $\varepsilon$ in a case of at most $m \cdot 4\left\lceil\log _{2} n\right\rceil$ strings comparing invocations.

As a second step, we construct a suffix array suf for $t$. Then, we consider an array $a$ of pairs $(l e n, i n d)$. One element of $a$ corresponds to one element of the suffix array suf. After that, we construct a segment tree st for $a$ and use len parameter of pair for maximum.

As a next step, we consider strings $s^{i}$ for $i \in\{1, \ldots m\}$. For each string $s^{i}$ we find the smallest index low and the biggest index high such that all suffixes $t\left[s u f_{j}, n\right]$ for low $\leq j \leq h i g h$ has $s^{i}$ as a prefix. We can use binary search for this action. Because of sorted order of suffixes in suffix array, all suffixes with the prefix $s^{i}$ are situated sequently. As a comparator for strings, we use COMPARE procedure. Let us present this action as a procedure $\operatorname{SeARChSEgment}(u)$ in Algorithm 2. The algorithm returns $(N U L L, N U L L)$ if no suffix of $t$ contains $u$ string as a prefix.

Then, we update values in the segment tree st by a pair $\left(\left|s^{i}\right|, i\right)$.

After processing all strings from $\left(s^{1}, \ldots, s^{m}\right)$, the array $a$ is constructed. We can construct long array using $a$ and the suffix array suf. We know that $i$-th element (len, ind) stores the longest possible string $s^{\text {ind }}$ that starts from $s u f_{i}$. It is almost definition of long array. So we can put $l_{\text {ong }} g_{\text {suf }}=i n d$, if $a_{i}=($ ind,len $)$.

Finally, we get the following Algorithm 3 for the text constructing from a dictionary problem.

Let us discuss properties of Algorithm 3.

Theorem 2. Algorithm 3 solves the text $t$ constructing from a dictionary $s=\left(s^{1}, \ldots, s^{m}\right)$ problem with $O\left(n+L+m(\log n)^{2}-\log \varepsilon\right)$ running time end error probability $\varepsilon$ for some $\varepsilon>0, n=|t|$ and $L=\left|s^{1}\right|+\cdots+\left|s^{m}\right|$. The running time is $O\left(n+L+m(\log n)^{2}\right)$ in a case of $\varepsilon=$ const.

Proof. The correctness of the algorithm follows from construction.

Let us discuss running time of the algorithm. Note, that $m \leq L=\sum_{j=1}^{m}\left|s^{j}\right|$ and $1 \leq\left|s^{j}\right| \leq n$ for $j \in\{1, \ldots, m\}$.

Due to results from Section 2.2, ComputeKI works with $O(n+\log p)$ running time. Let us convert this statement.

$$
\begin{gathered}
O(n+\log p)=O(n+\log (n \cdot \alpha / \varepsilon))= \\
=O(n+\log n+\log \alpha-\log \varepsilon)=O(n+\log \alpha-\log \varepsilon)= \\
=O\left(n+\log \left(m \cdot 4\left\lceil\log _{2} n\right\rceil^{2}\right)-\log \varepsilon\right)=O(n+\log m+\log \log n-\log \varepsilon)= \\
=O(n+\log m-\log \varepsilon)
\end{gathered}
$$

Due to results from Section 2.2, ComputePrefixRollingHashes works in linear running time. Therefore, all invocations of ComputePrefixRollingHashes procedure works in $O\left(n+\sum_{j=1}^{m}\left|s^{j}\right|\right)=O(n+L)$ running time. 


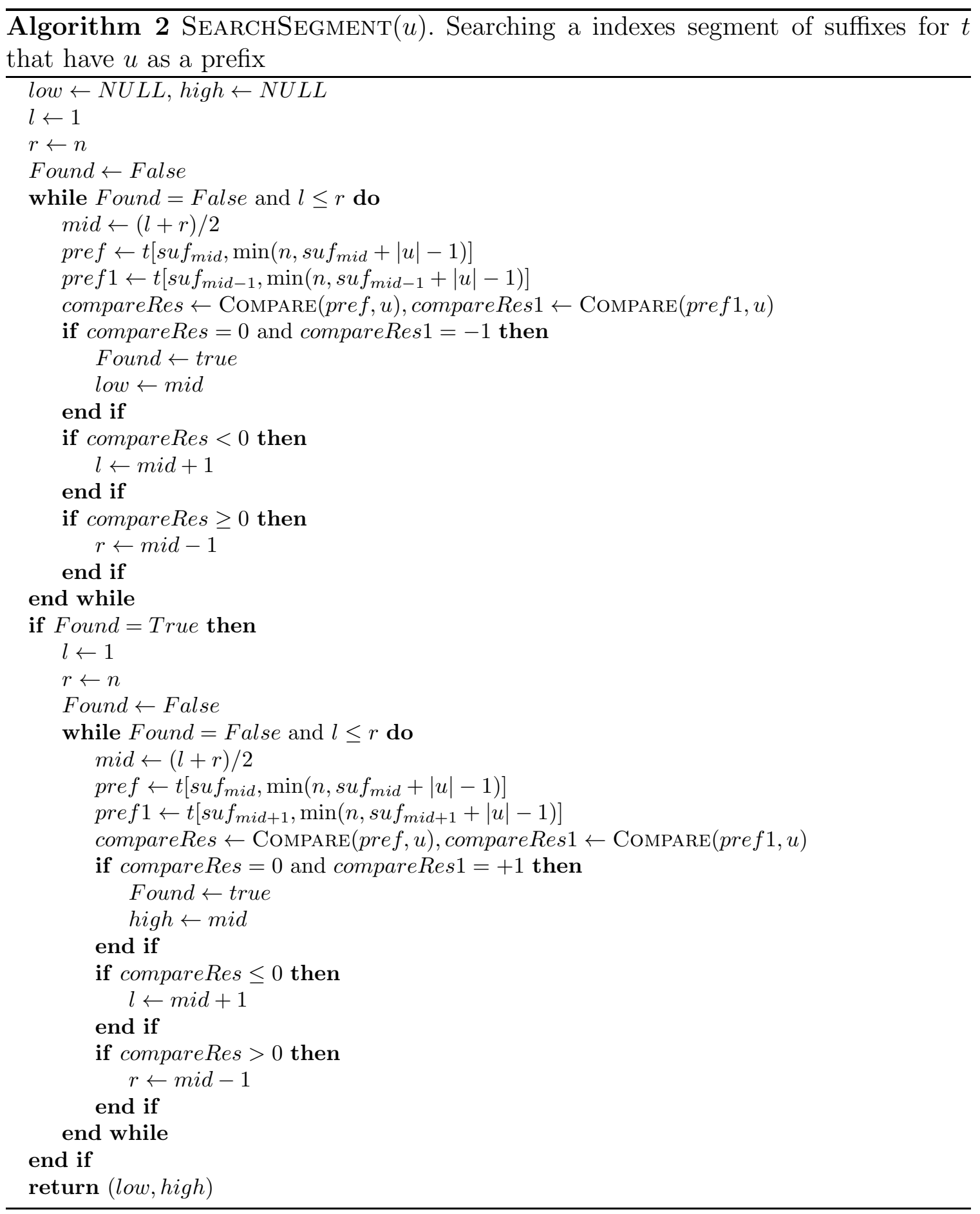




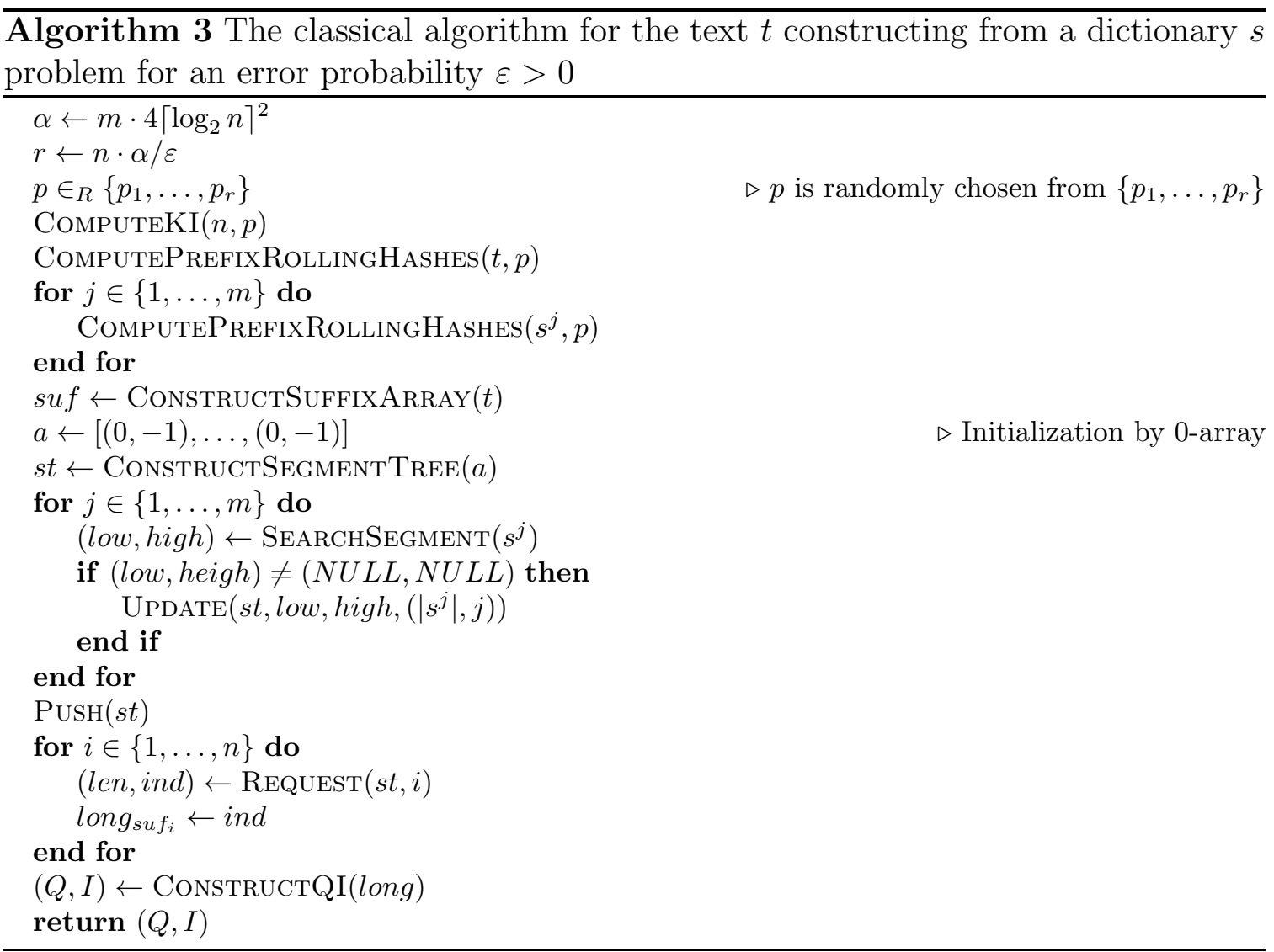

Due to Lemma 1, ConstructSuffixArray works in $O(n)$ running time. Initializing of $a$ does $O(n)$ steps. Due to results from Section 2.3, ConstructSegmentTree works in $O(n)$ running time.

SEARChSEgment procedure invokes Compare procedure $O(\log n)$ times due to binary search complexity. COMPARE procedure works in $O(\log n)$ running time. Therefore, SearchSegment works in $O\left((\log n)^{2}\right)$ running time. Due to results from Section 2.3, UpDATE procedure works in $O(\log n)$ running time. Hence, the total complexity of processing all strings from the dictionary $s$ is $O\left(m \cdot\left((\log n)^{2}+\log n\right)\right)=$ $O\left(m \cdot(\log n)^{2}\right)$.

The invocation of PusH works in $O(n)$ running time due to results from Section 2.3. The invocation of REQUEST works in $O(1)$ running time because we do not invoke UpDATE after Push. Therefore, constructing of the array long takes $O(n)$ steps.

The running time of ConstructQI is $O(n)$ because we pass each element only once.

So, the total complexity of the algorithm is

$$
\begin{gathered}
O\left(n+\log m-\log \varepsilon+n+L+n+n+n+m(\log n)^{2}+n+n+n\right)= \\
=O\left(n+L+m(\log n)^{2}-\log \varepsilon\right) .
\end{gathered}
$$

Let us discuss the error probability. We have $4 \cdot m\left\lceil\log _{2} n\right\rceil$ invocations of COMPARE procedure. Each invocation of COMPARE procedure compares rolling hashes at most $\left\lceil\log _{2} n\right\rceil$ times. Due to results from Section 2.2, if we compare strings of length at most $n$ using rolling hash $4 \cdot m\left\lceil\log _{2} n\right\rceil^{2}$ times and choose $p$ from $r$ primes, then we 
get error probability at most $\varepsilon$.

\subsection{A Quantum Algorithm}

Firstly, let us discuss a quantum subroutine. There is a quantum algorithm for comparing two strings in a lexicographical order with the following property:

Lemma 3 ([10]). There is a quantum algorithm that compares two strings of length $k$ in lexicographical order with query complexity $O(\sqrt{k} \log \gamma)$ and error probability $O\left(\frac{1}{\gamma^{3}}\right)$ for some positive integer $\gamma$.

Let QCOMPARE_STRINGS_BASE $(u, v, l)$ be a quantum subroutine for comparing two strings $u$ and $v$ of length $l$ in lexicographical order. We choose $\gamma=m \log n$. In fact, the procedure compares prefixes of $u$ and $v$ of length $l$. QCOMPARE_STRINGS_BASE $(u, v, l)$ returns 1 if $u>v$; it returns -1 if $u<v$; and it returns 0 if $u=v$.

Next, we use a QCompare $(u, v)$ that compares $u$ and $v$ string in lexicographical order. Assume that $|u|<|v|$. Then, if $u$ is a prefix of $v$, then $u<v$. If $u$ is not a prefix of $v$, then the result is the same as for QCOMPARE_STRINGS_BASE $(u, v,|u|)$. In the case of $|u|>|v|$, the algorithm is similar. The idea is presented in Algorithm 4.

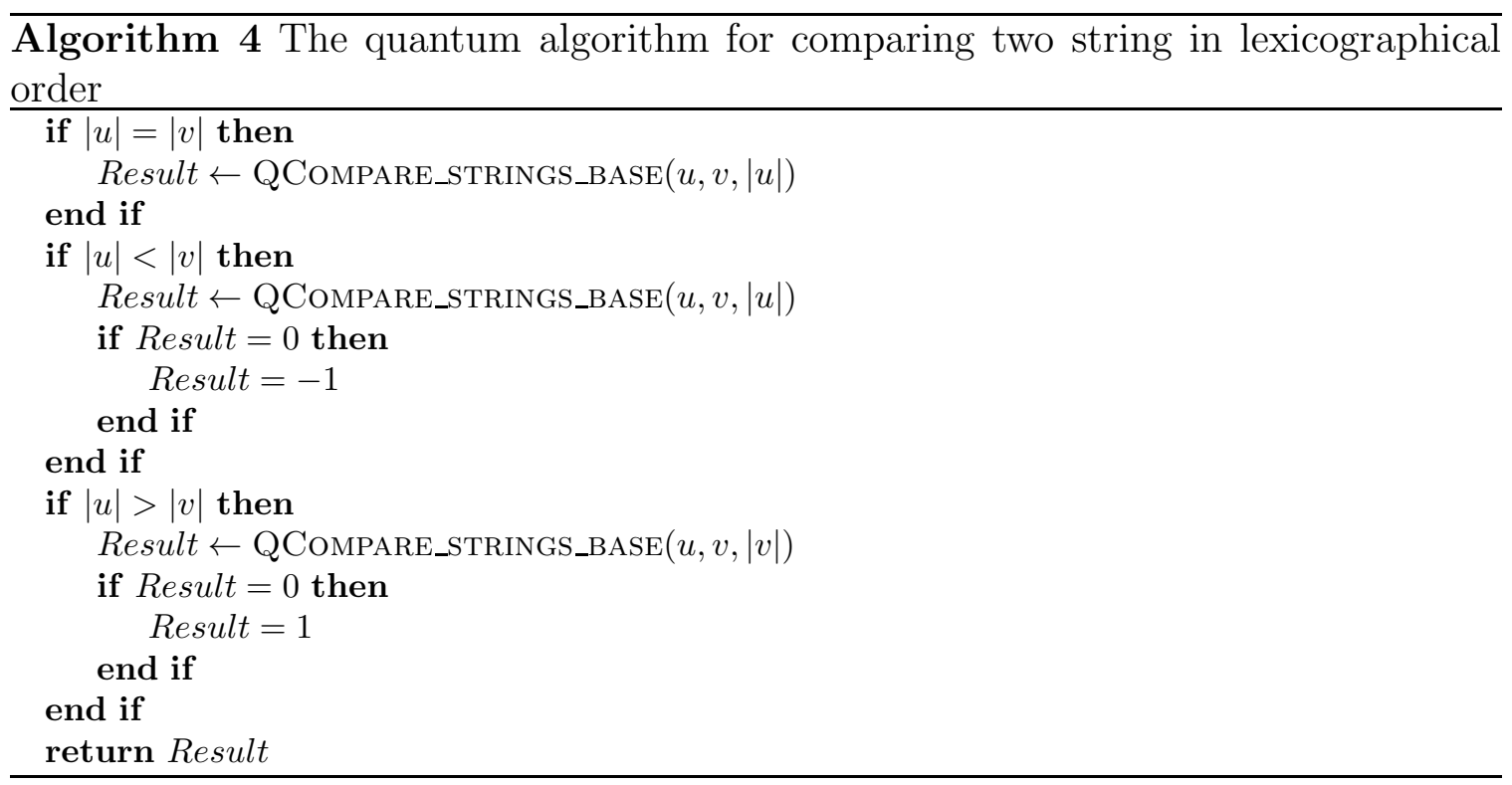

Let us present a quantum algorithm for the text constructing form a dictionary problem. For the algorithm, we use the same idea as in the classical case, but we replace COMPARE that uses the rolling hash function by QCOMPARE. In that case, we should not construct rolling hashes. Let QSEARCHSEGMENT be a quantum counterpart of SEARCHSEgment that uses QCOMPARE.

The quantum algorithm is presented as Algorithm 5.

Let us discuss properties of Algorithm 5.

Theorem 4. Algorithm 5 solves the text $t$ constructing from a dictionary $s=\left(s^{1}, \ldots, s^{m}\right)$ problem in $O(n+\log n \cdot(\log m+\log \log n) \cdot \sqrt{m \cdot L})$ running time end error probability $O\left(\frac{1}{m+\log n}\right)$. 


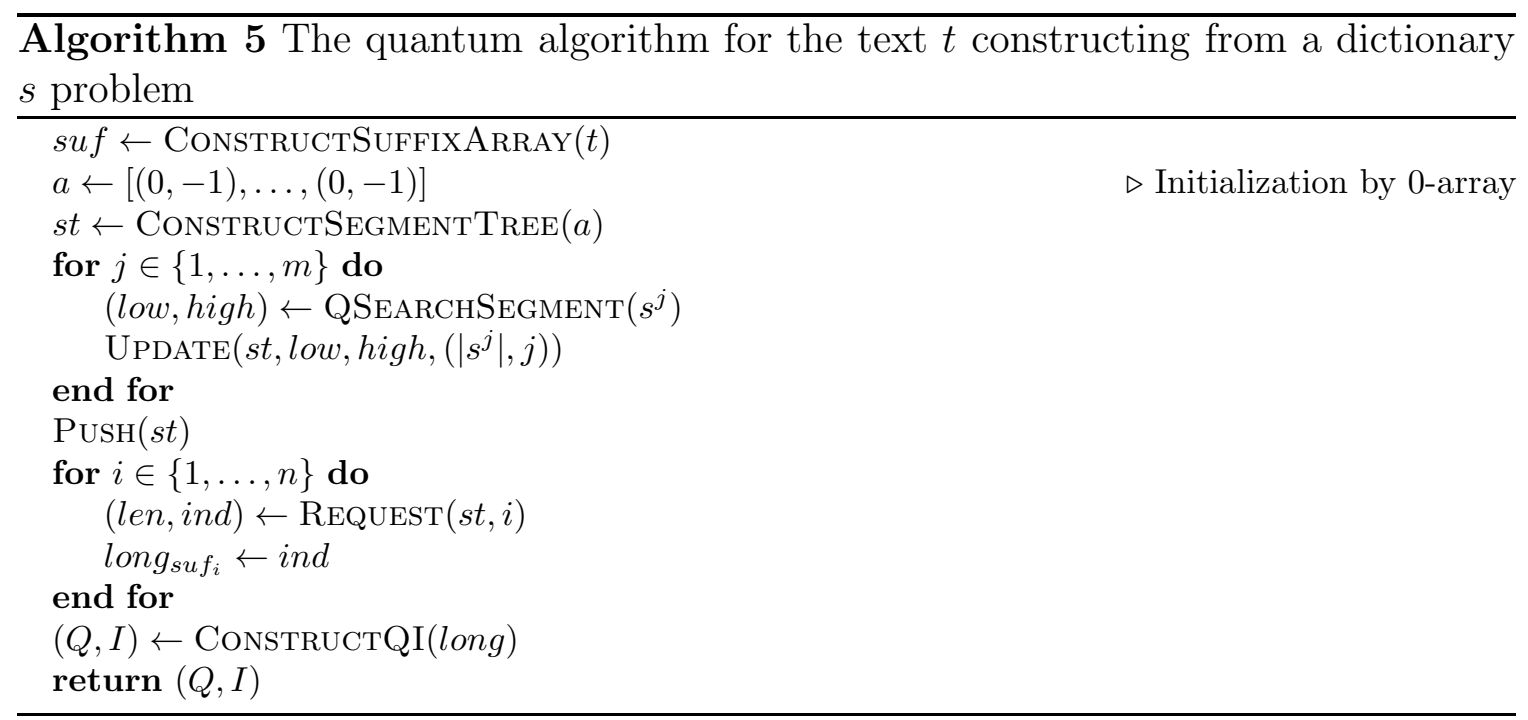

Proof. The algorithm does almost the same actions as the classical counterpart. That is why the correctness of the algorithm follows from Theorem 2.

Let us discuss the running time. Due to Theorem 2, the running time of the procedure ConstructSuffixARrAY is $O(n)$, the running time of the procedure ConstructSegmentTree is $O(n)$, the running time of the procedure Push is $O(n)$, the running time of the array long construction is $O(n)$, the running time of ConstructQI is $O(n)$.

Due to Lemma 3, the running time of QCOMPARE for $s^{j}$ is $O\left(\sqrt{\left|s^{j}\right|}(\log m+\right.$ $\log \log n)$ ). The procedure QSEARChSEgmEnT invokes QCompare procedure $O(\log n)$ times for each string $s^{1}, \ldots s^{m}$. So, the complexity of processing all strings from $s$ is

$$
O\left(\log n \cdot(\log m+\log \log n) \cdot \sum_{j=1}^{m} \sqrt{\left|s^{j}\right|}\right)
$$

Let us use the Cauchy-Bunyakovsky-Schwarz inequality and $L=\sum_{j=1}^{m}\left|s^{j}\right|$ equality for simplifying the statement.

$\leq O\left(\log n \cdot(\log m+\log \log n) \cdot \sqrt{m \sum_{j=1}^{m}\left|s^{j}\right|}\right)=O(\log n \cdot(\log m+\log \log n) \cdot \sqrt{m \cdot L})$.

The total running time is

$$
\begin{gathered}
O(n+n+n+\log n \cdot(\log m+\log \log n) \cdot \sqrt{m \cdot L})= \\
=O(n+\log n \cdot(\log m+\log \log n) \cdot \sqrt{m \cdot L})
\end{gathered}
$$

Let us discuss the error probability. The algorithm invokes QCOMPARE procedure $2 m\left\lceil\log _{2} n\right\rceil \leq 2 m\left(1+\log _{2} n\right)$ times. The success probability is

$$
O\left(\left(1-\frac{1}{(m \log n)^{3}}\right)^{2 m\left(1+\log _{2} n\right)}\right)=O\left(\frac{1}{m \log n}\right) .
$$




\section{Lower Bound}

Let us discuss the lower bound for the running time of classical algorithms.

Theorem 5. Any randomized algorithm for the text $t$ constructing from a dictionary $s=\left(s^{1}, \ldots, s^{m}\right)$ problem works in $\Omega(n+L)$ running time, where $L=\left|s^{1}\right|+\cdots+\left|s^{m}\right|$.

Proof. Assume $L>n$. Let us consider $t=\left(t_{1}, \ldots, t_{n}\right)$ such that $t_{\lfloor n / 2\rfloor}=1$ and $t_{i}=0$ for all $i \in\{1, \ldots, n\} \backslash\{\lfloor n / 2\rfloor\}$.

Let $\left|s^{i}\right| \leq n / 2$ for each $i \in\{1, \ldots, m\}$. Note, that in a general case $\left|s^{i}\right| \leq n$. Therefore, we reduce the input data only at most twice. Assume that we have two options:

- all $s^{i}$ contains only $0 \mathrm{~s}$;

- there is $z$ such that we have two conditions:

- all $s^{i}$ contains only 0s, for $i \in\{1, \ldots, m\} \backslash\{z\}$;

- for $j_{0}<\left|s^{z}\right|, s_{j_{0}}^{z}=1$ and $s_{j}^{z}=0$ for $j \in\left\{1, \ldots,\left|s^{z}\right|\right\} \backslash\left\{j_{0}\right\}$.

In a case of all $0 \mathrm{~s}$, we cannot construct the text $t$. In a case of existing 0 in the first half, we can construct $t$ by putting $s^{z}$ on the position $j_{0}-\lfloor n / 2\rfloor+1$ and we get 1 of the required position. Then, we complete $t$ by other 0 -strings.

Therefore, the solution of the problem is equivalent to the search of 1 in unstructured data of size $L$. The randomized complexity of this problem is $\Omega(L)$ due to [4].

Assume $L<n$. Let $m=1,\left|s^{1}\right|=1$ and $s_{1}^{1}=1$. Assume that we have two options:

$-t$ contains only $1 \mathrm{~s}$;

- there is $g$ such that $t_{g}=0$ and $t_{j}=1$ for all $j \in\{1, \ldots, n\} \backslash\{g\}$.

In the first case, we can construct $t$ from $s$. In the second case, we cannot do it.

Here the problem is equivalent to search 0 among $n$ symbols of $t$. Therefore, the problem's randomized complexity is $\Omega(n)$.

Finally, the total complexity is $\Omega(\max (n, L))=\Omega(n+L)$.

\section{References}

1. F. Ablayev, M. Ablayev, J. Z. Huang, K. Khadiev, N. Salikhova, and D. Wu: On quantum methods for machine learning problems part i: Quantum tools. Big Data Mining and Analytics, 3(1) 2019, pp. 41-55.

2. A. AmBAINIS: Understanding quantum algorithms via query complexity. arXiv:1712.06349, 2017.

3. S. Behjati AND P. S. Tarpey: What is next generation sequencing? Archives of Disease in Childhood - Education and Practice, 98(6) 2013, pp. 236-238.

4. C. H. Bennett, E. Bernstein, G. Brassard, and U. Vazirani: Strengths and weaknesses of quantum computing. SIAM journal on Computing, 26(5) 1997, pp. 1510-1523.

5. T. H. Cormen, C. E. Leiserson, R. L. Rivest, and C. Stein: Introduction to Algorithms, McGraw-Hill, 2001.

6. R. De Wolf: Quantum computing and communication complexity, 2001.

7. R. FreIvalds: Fast probabilistic algorithms, in Mathematical Foundations of Computer Science 1979, vol. 74 of LNCS, 1979, pp. 57-69. 
8. S. JoRDAN: Bounded error quantum algorithms zoo, https://math.nist.gov/quantum/zoo.

9. R. M. KARP AND M. O. RABIN: Efficient randomized pattern-matching algorithms. IBM journal of research and development, 31(2) 1987, pp. 249-260.

10. K. KHADIEV AND A. ILIKAEv: Quantum algorithms for the most frequently string search, intersection of two string sequences and sorting of strings problems, in International Conference on Theory and Practice of Natural Computing, 2019, pp. 234-245.

11. K. Khadiev, D. Kravchenko, And D. Serov: On the quantum and classical complexity of solving subtraction games, in Proceedings of CSR 2019, vol. 11532 of LNCS, 2019, pp. 228-236.

12. K. KHADIEV AND L. SAFinA: Quantum algorithm for dynamic programming approach for dags. applications for zhegalkin polynomial evaluation and some problems on dags, in Proceedings of UCNC 2019, vol. 4362 of LNCS, 2019, pp. 150-163.

13. A. LaAksonen: Guide to Competitive Programming, Springer, 2017.

14. Z. LI, J. Li, AND H. HuO: Optimal in-place suffix sorting, in String Processing and Information Retrieval, Cham, 2018, Springer International Publishing, pp. 268-284.

15. U. Manber AND G. Myers: Suffix arrays: A new method for on-line string searches, in Proceedings of the First Annual ACM-SIAM Symposium on Discrete Algorithms, SODA 90, Society for Industrial and Applied Mathematics, 1990, p. 319327.

16. A. Montanaro: Quantum pattern matching fast on average. Algorithmica, 77(1) 2017, pp. 1639 .

17. E. W. Myers, G. G. Sutton, A. L. Delcher, I. M. Dew, D. P. Fasulo, M. J. Flanigan, S. A. Kravitz, C. M. Mobarry, K. H. Reinert, K. A. Remington, et Al.: A wholegenome assembly of drosophila. Science, 287(5461) 2000, pp. 2196-2204.

18. M. A. Nielsen And I. L. ChuAng: Quantum computation and quantum information, Cambridge univ. press, 2010.

19. H. RAMESH AND V. VinAY: String matching in $o(\sqrt{n}+\sqrt{m})$ quantum time. Journal of Discrete Algorithms, 1(1) 2003, pp. 103-110. 